To appear in Vehicle System Dynamics

Vol. 00, No. 00, Month 20XX, 1-15

\title{
Flow-field guided steering control for rigid autonomous ground vehicles in low-speed manoeuvring
}

\author{
Mengxuan Song ${ }^{\mathrm{a}}$, Nan Wang ${ }^{\mathrm{a}}$, Timothy Gordon ${ }^{\mathrm{b}}$ and Jun Wang ${ }^{\mathrm{a} *}$ \\ ${ }^{a}$ Department of Control Science and Engineering, Tongji University, Shanghai, PR China; \\ ${ }^{b}$ University of Lincoln, Lincoln, UK
}

(Received 00 Month 20XX; accepted 00 Month 20XX)

\begin{abstract}
This paper studies the low-speed manoeuvring problem for autonomous ground vehicles operating in complex static environments. Making use of the intrinsic property of a fluid to naturally find its way to an outflow destination, a novel guidance method is proposed. In this approach, a reference flow field is calculated numerically through Computational Fluid Dynamics, based on which both the reference path topology and the steering reference to achieve the path are derived in a single process. Steering control considers three constraints: obstacle and boundary avoidance, rigidity of the vehicle, plus the non-holonomic velocity constraints due to the steering system. The influences of the parameters used during the flow field simulation and the control algorithm are discussed through numerical cases. A divergency field is defined to evaluate the quality of the flow field in guiding the vehicle. This is used to identify any problematic branching features of the flow, and control is adapted in the neighbourhood of such branching features to resolve possible ambiguities in the control reference. Results demonstrate the effectiveness of the method in finding smooth and feasible motion paths, even in complex environments.
\end{abstract}

Keywords: autonomous vehicles; motion control; flow field; computational fluid dynamics

\section{Introduction}

This paper addresses the guidance and steering control problem of ground vehicles that require precise low-speed manoeuvring in environments with complex borders and obstacles, e.g. in autonomous freight operations where large vehicles operate within a limited motion space. We face three typical technical challenges in such situations: (1) choosing an optimal (or at least feasible) path topology, i.e. decision making about which turns to make in order to reach the goal; (2) constructing a suitable (feasible and smooth) reference path; (3) applying steering control to follow the reference path, while respecting collision avoidance constraints with the boundaries and the obstacles. The problem becomes more complex and difficult when manoeuvring cars or trucks in narrow spaces, taking account of non-holonomic constraints from the wheel motions. This paper aims at presenting and demonstrating a concept that the manoeuvring of a rigid autonomous ground vehicle can be guided by a flow field. A simple kinematic bicycle model will be used for the vehicle during the simulation. Therefore, for low-speed manoeuvres, zero sideslip angles may be assumed.

\footnotetext{
${ }^{*}$ Corresponding author. Email: junwang@tongji.edu.cn
} 
Various navigation methods have been developed on this topic. Conventional graph search algorithms, such as Dijkstra [1], $\mathrm{A}^{*}[2]$ and $\mathrm{D}^{*}[3]$ algorithms, search the global map for a feasible path. However, these algorithms do only the path construction, without considering the limits such as how sharp the subject can turn. Applying this type of algorithm alone is not practical for non-holonomic systems.

Artificial Intelligent (AI) algorithms including fuzzy logic algorithm [4], genetic algorithm [5] and neural networks [6] have been successfully implemented in the path planning problem. The AI based methods are able to handle constraints of vehicle kinematics and the environment. But the evolutional algorithms such as the genetic algorithm do not guarantee to find the optimal solution, and the neural network cannot work perfectly as we expected. These kinds of methods may fail to obtain a solution sometimes when there actually are feasible solutions.

Another type of conventional approach is the Artificial Potential Field (APF) method [7], proposed to deal with geometrical constraints by introducing potential functions. The APF method is capable of providing a smooth and collision-free paths, but has the drawback of that the subject can become trapped in local minima of the potential field, rendering it inappropriate for environments with concave boundaries.

Much research has also been devoted to the sampling based planners such as the Rapidly-exporing Randomised Tree (RRT) method [8] and the probabilistic road map method [9]. The key disadvantage of the sampling based path planning methods is that the probabilistic completeness is achieved only when the number of samples approaches infinity, i.e. finding a feasible path is not guaranteed because infinite sampling cannot be achieved in practice. Besides, the current methods of this kind do not consider nonholonomic constraints, and not particularly suitable for such systems.

For a non-holonomic system such as a self-driving car, the kinematic constraints can render a path infeasible, and the feasibility highly depends on the configuration of the vehicle. For example, if the rear wheels are steerable, and the vehicle can do in-place rotations, more paths will become feasible. This paper considers a novel and more direct technique inspired by the physics of fluid motions and the previous work on high speed motion control $[10,11]$. The aim is to define and evaluate a path planning and steering control methodology that can be efficient, modular, open to diagnostic scrutiny and also seamless across different speed ranges. In this paper, we introduce, test and discuss the underlying concept, restricting our attention to low-speed manoeuvring.

\section{Methodology}

Natural phenomena, including optics and fluid flow, have inherent abilities to find feasible and optimal paths in a given environment. Although a fluid has no inherent objective, it does have the ability to find pathways through a bounded environment from a prescribed inflow boundary to any available outflow boundaries. Although fluid flow does not exactly match the purpose of vehicle guidance, desirable features of the fluid flow can motivate and partially solve motion control problems for autonomous vehicles. In the present consideration, the velocity field of the fluid flow (or flow field for short) provides a collection of ubiquitous signposts throughout the domain. With appropriately designed algorithms, the preferred steering angle of the vehicle can be calculated directly from the flow field in the vicinity of the vehicle, and the outcome can possibly lead the vehicle to the designated target without the need for traditional path-following control strategies. With the help of the local flow field, the vehicle may automatically avoid collision with boundaries and obstacles, and eventually reach the target. 
Based on this concept, the proposed control framework consists of three layers. The first is the strategic layer, where the general global mission is chosen, i.e. selecting the desired initial and terminal positions for the vehicle within some localised region. The second layer is the tactical layer, which will produce a flow field that will act as the guidance to the vehicle motion. The Computational Fluid Dynamics (CFD) method is introduced to solve the flow field in the environment of given boundaries and obstacles. In this paper, ANSYS FLUENT is used to perform the CFD calculations. The third layer is the control layer, where steering control is implemented with information obtained from the flow field. Note that in the present study, the magnitude of the fluid velocity is irrelevant, but only its direction matters.

\subsection{Flow field simulation}

Fluid motions include various types of phenomena, including turbulence, compressibility, phase change, etc. Currently since the flow field here is to provide reference or guidance to the motion control of the vehicle, it should have the following particular features. (1) Completeness should be guaranteed, meaning that the fluid must always reach the destination no matter where it starts; (2) Smoothness of the streamlines, which makes it easier for the vehicle to follow; (3) Random turbulent fluctuations should be eliminated since this would not be beneficial to smooth and stable control. Considering that the flow field here is not required to reflect all aspects of real-world fluid motion, a number of preconditions are imposed to obtain the flow field.

The borders of the fluid domain are set exactly the same as the geometry of the boundaries and obstacles that the vehicle may encounter. An inlet of the fluid domain is located immediately behind the vehicle at its defined initial state, while an outlet is located in front of vehicle at its expected final state. Since we are dealing with the ground vehicle on a horizontal surface, the two-dimensional laminar Navier-Stokes (NS) equations without gravity are used. Letting $\mathbf{u}(\mathbf{x})$ be the velocity vector of the fluid flow at Position $\mathbf{x}$, the governing equations that determine the flow field are

$$
\begin{gathered}
\nabla \mathbf{u}=0 \\
\rho \mathbf{u} \cdot \nabla \mathbf{u}=-\nabla p+\nabla \cdot(\mu \nabla \mathbf{u})
\end{gathered}
$$

where $\rho$ is the density of the fluid, $p$ is the pressure, and $\mu$ is the dynamic viscosity. In Equation 2, the pressure gradient term $\nabla p$ cannot be chosen arbitrarily. Instead, it is solved so that the velocity field $\mathbf{u}$ satisfies the mass conservation law in Equation 1. Since the absolute magnitude of $p$ is not our concern in this study, the characteristics of the velocity field $\mathbf{u}$ are only determined by the ratio of $\rho$ and $\mu$, rather than the absolute values of $\rho$ and $\mu$. For simplification, physical properties of the fluid are set as constants according to the normal state of air, where $\rho$ and $\mu$ are valued $1.225 \mathrm{~kg} / \mathrm{m}^{3}$ and $1.7894 \times 10^{-5} \mathrm{~kg} /(\mathrm{m} \cdot \mathrm{s})$, respectively.

In order to achieve completeness, the borders of the boundaries and all obstacles are set as impenetrable and no-slip walls. Letting $U$ and $L$ be the characteristic fluid speed and the characteristic length respectively, the Reynolds number is defined by $\operatorname{Re}=\rho U L / \mu$. Small values of Re helps to let the flow field stay laminar, vortices and recirculation areas can be excluded in some extent, producing more streamlines that lead to the outlet. By using these settings, the features mentioned in the first paragraph of this section can be implemented, the flow field is able to provide information for the ground 


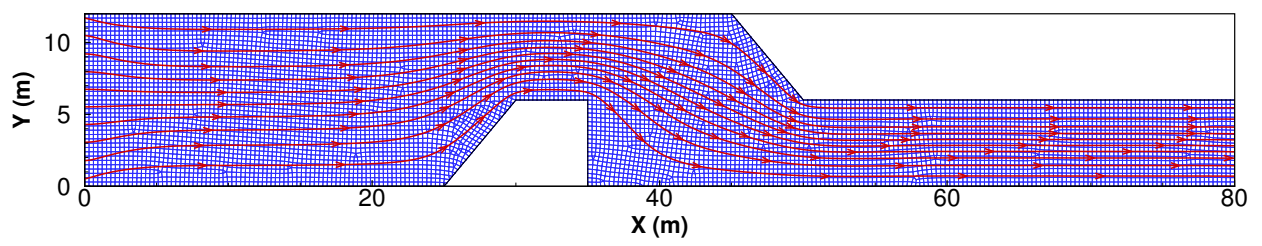

(a) Inlet speed is $10^{-5} \mathrm{~m} / \mathrm{s}$

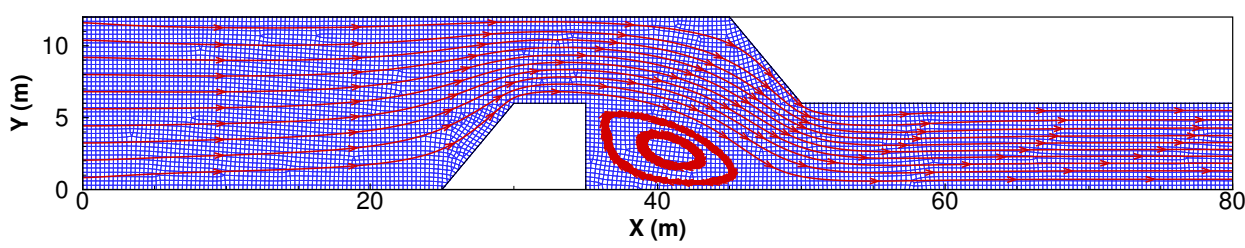

(b) Inlet speed is $10^{-3} \mathrm{~m} / \mathrm{s}$

Figure 1. An example of discretised mesh and calculated streamlines

vehicle, guiding it back on track when it has been deviated from its desired course. In the following examples, a constant velocity vector is assigned to the inlet boundary, with its magnitude at $10^{-5} \mathrm{~m} / \mathrm{s}$, and its direction normal to the inlet boundary. The outlet region is assigned as an outflow boundary without reflux.

To solve the flow field numerically, the domain is discretised with mixed quadrilateral grids and triangular grids with similar sizes and approximately uniform distribution. The mesh size is around $0.3 \mathrm{~m}$. Mesh generation is done with ANSYS ICEM CFD 15, and the flow field is solved with the steady laminar model using ANSYS Fluent 15. An example of the discretised mesh and the calculated flow field is shown in Figure 1(a), where blue grid lines represent the borders of the cells in the mesh. The simulated flow enters the domain at $X=0 \mathrm{~m}$ and exists at $X=80 \mathrm{~m}$. The calculated flow field contains a velocity vector at every cell in the mesh. Several streamlines are generated from the flow field and plotted with red curves. Because of mass conservation, every portion of fluid that enters the domain must leave through the outlet. This demonstrates the search capability of the proposed method. For comparison, a simulation with inlet speed at $10^{-3} \mathrm{~m} / \mathrm{s}$ is performed. With fluid speed at this level, the Reynolds number is high enough so that turbulence models are essential. Here we introduce the standard $k$ - $\varepsilon$ model to calculate the turbulent effect. The result is plotted in Figure 1(b). It is seen that even with a recirculation area behind the obstacle, the streamlines originated at the inlet region can still reach the outlet region, while the fluid inside the recirculation area remains within that area. If the flow field with vortices or recirculation areas is used to guide the vehicle, and some unpredictable disturbance drives the vehicle into a recirculation area accidentally, it may become trapped, and hence the low Reynolds number condition is desirable.

\subsection{Guiding the vehicle}

To conduct preliminary test and validation for the proposed flow-field guidance method, the simplest kinematic bicycle model is employed to consider and simulate the movement of the vehicle. The proposed control method operates in a vehicle-based local coordinate 


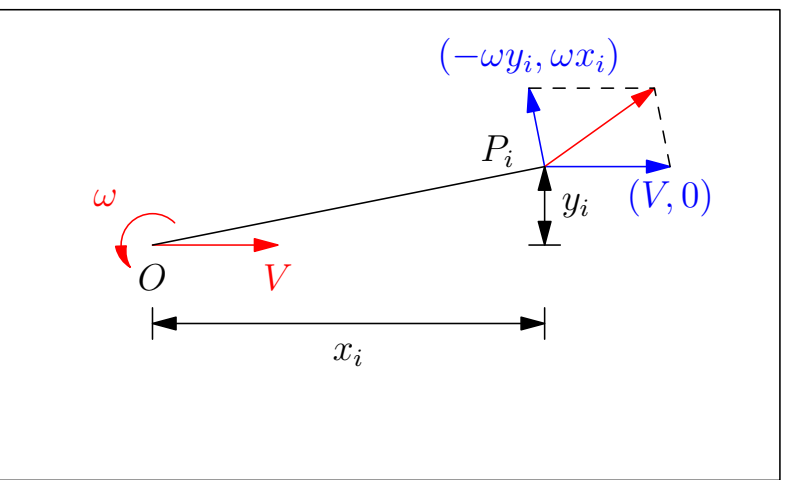

Figure 2. Velocity of a point on the vehicle body

system, where the point of origin is located at the centre of the rear axle. The longitudinal direction and the lateral direction to the left are designated as the $x$ axis and the $y$ axis respectively (ISO convention). This is convenient as the point of origin serves as a pivot point, with zero sideslip at the un-steered rear axle, and the velocity vector always lies along the $x$ axis. If $V$ is the speed of the pivot point relative to the ground, and $\omega$ is the yaw rate of the vehicle, the velocity vector at any point $\left(x_{i}, y_{i}\right)$ on the vehicle body is $\left(V-\omega y_{i}, \omega x_{i}\right)$, as shown in Figure 2, where blue arrows represent velocity components. Note that $\omega$ is constrained by a limitation of the minimum turning radius $R_{\min }$ :

$$
|\omega| \leq \frac{V}{R_{\min }}
$$

At each time step, the vehicle body covers $N$ nodes of the domain ( $N$ may not be constant through the process, because the discretised mesh can not be absolutely uniform), each of which has a velocity vector of the fluid, e.g. $\left(u_{i}, v_{i}\right)$ for Point $P_{i}$. The proposed control strategy aims at determining the value of $\omega$ that can make the velocity of the vehicle align with the fluid velocity at corresponding position as much as possible. Equating the velocity ratios, we obtain, for a single Point $P_{i}$ :

$$
\left(u_{i} x_{i}+v_{i} y_{i}\right) \omega_{i}=v_{i} V
$$

and $\omega_{i}$ can be calculated directly if only a single reference point is used. However, the vehicle is a rigid body, and the velocity directions are unable to match with the flow field at all points simultaneously. To obtain an optimal match for a set of reference points $P_{i}$, $(i=1, \ldots, n)$, the least square method is utilised to calculate $\omega$ for the vehicle. Defining

$$
\mathbf{a}=\left(\begin{array}{c}
u_{1} x_{1}+v_{1} y_{1} \\
u_{2} x_{2}+v_{2} y_{2} \\
\vdots \\
u_{n} x_{n}+v_{n} y_{n}
\end{array}\right), \quad \mathbf{b}=\left(\begin{array}{c}
v_{1} V \\
v_{2} V \\
\vdots \\
v_{n} V
\end{array}\right)
$$

the optimal value of $\omega$ is calculated through

$$
\hat{\omega}=\left(\mathbf{a}^{\mathrm{T}} \mathbf{a}\right)^{-1} \mathbf{a}^{\mathrm{T}} \mathbf{b}
$$

If $\hat{\omega}$ does not satisfy the constraint described in Equation 3, the final outcome of $\omega$ will 


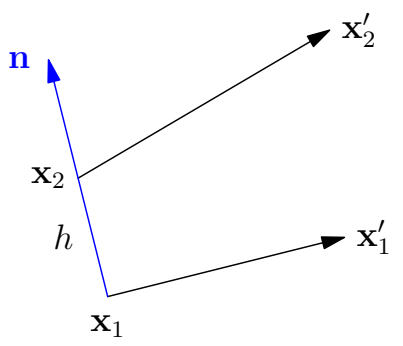

Figure 3. Sketch of the divergency

be the corresponding boundary value $\left(V / R_{\min }\right.$ or $\left.-V / R_{\min }\right)$.

\subsection{Quality of the flow field}

Within the area of the flow field covered by the vehicle body, if all fluid particles are moving in the same direction, the vehicle gets perfect guidance. If the fluid is converging or diverging, e.g. in a narrowing tunnel or in a widening tunnel, the nodes in the flow field give different indications on the direction. The converging flow will not cause serious problems on the guidance of the vehicle, since the fluid will eventually reach adjacent destinations. However, the diverging flow confuses the vehicle about which direction to go to. In other word, the flow field is in poor quality. Therefore, a divergency field is defined to evaluate the quality of the flow field.

Consider a point in the flow field, denoted as $\mathbf{x}_{1}$, as depicted in Figure 3 . The velocity at $\mathbf{x}_{1}$ is $\mathbf{u}_{1}$. Let $\mathbf{n}$ as a unit vector normal to $\mathbf{u}_{1}$. A second point $\mathbf{x}_{2}$ is located along the direction of $\mathbf{n}$ with a small distance $h$ to $\mathbf{x}_{1}$. Hence $h=\mathbf{n} \cdot\left(\mathbf{x}_{2}-\mathbf{x}_{1}\right)$. After a small time $\Delta t$, fluid particles located at these two points will respectively move to

$$
\mathbf{x}_{1}^{\prime}=\mathbf{x}_{1}+\mathbf{u}_{1} \Delta t, \quad \mathbf{x}_{2}^{\prime}=\mathbf{x}_{2}+\mathbf{u}_{2} \Delta t
$$

The distance of the two fluid particles along the direction of $\mathbf{n}$ becomes

$$
h^{\prime}=\mathbf{n} \cdot\left(\mathbf{x}_{2}^{\prime}-\mathbf{x}_{1}^{\prime}\right)=\mathbf{n} \cdot\left[\left(\mathbf{x}_{2}-\mathbf{x}_{1}\right)+\left(\mathbf{u}_{2}-\mathbf{u}_{1}\right) \Delta t\right]
$$

When $h \rightarrow 0$, it is obtained that

$$
\mathbf{u}_{2}-\mathbf{u}_{1}=h \mathbf{n} \cdot \nabla \mathbf{u}
$$

where $\nabla \mathbf{u}$ is the gradient tensor of the vector field $\mathbf{u}$ at $\mathbf{x}_{1}$. The divergency at $\mathbf{x}_{1}$ is then defined as

$$
\begin{aligned}
\delta & =\lim _{h \rightarrow 0} \frac{h^{\prime}-h}{h \Delta t} \\
& =\lim _{h \rightarrow 0} \frac{\mathbf{n} \cdot\left(\mathbf{u}_{2}-\mathbf{u}_{1}\right)}{h} \\
& =\mathbf{n} \cdot(\mathbf{n} \cdot \nabla \mathbf{u})
\end{aligned}
$$

Since $h$ is always positive, if the flow is diverging, $h^{\prime}>h$, and $\delta$ is positive. If the flow is converging, $h^{\prime}<h$, and $\delta$ is negative. If the flow is neither converging nor diverging, 
$h^{\prime}=h$ and $\delta=0$. The value of $\delta$ can be calculated throughout the domain, forming a "divergency field", which will be plotted as filled contours in the examples below.

\subsection{Simulation of the vehicle motion}

With the control method defined, the motion of the vehicle in the environment can be simulated by integrating the motion within an inertial coordinate system. Vehicle states are: the global position $(X, Y)$, the global yaw angle $\theta$, the speed of the pivot point relative to the ground $V$, and the yaw rate relative to the ground $\omega$. Since the present study is focused on the low-speed manoeuvring problem, effects caused by acceleration are not considered, and the magnitude of $V$ can be chosen arbitrarily and it does not influence the outcome of the simulated path. Also, the yaw rate $\omega$, which is determined by the steering angle of the steering wheel, can be considered to be directly affected by the input. Therefore the model to simulate the motion of the vehicle in the global coordinate system is simplified as

$$
\dot{X}=V \cos \theta, \quad \dot{Y}=V \sin \theta, \quad \dot{\theta}=\omega
$$

\section{Simulation results and discussion}

The proposed control method and the simulation model are implemented with Python codes. The parameters used in the vehicle model are based on an MGGS 1.6T experimental vehicle from the Tongji VeCaN Lab. The vehicle is $4.500 \mathrm{~m}$ long and $1.855 \mathrm{~m}$ wide. The lengths of the vehicle body that protrudes beyond the front axle and the rear axle are $0.954 \mathrm{~m}$ and $0.896 \mathrm{~m}$, respectively. The minimum turning radius of this vehicle is $4.944 \mathrm{~m}$.

\subsection{Test scenarios}

Four typical scenarios are designed to test the proposed method:

(1) A double-lane-change scenario, as sketched in Figure 4. The vehicle needs to change to the left lane and then back to the right lane.

(2) A left-turn at a intersection, as sketched in Figure 5. The vehicle enters the intersection along the $Y+$ direction, and needs to exit along the $X$ - direction.

(3) A u-turn scenario, as sketched in Figure 6. The vehicle needs to perform a u-turn and to exit along the opposite direction.

(4) A simple maze scenario with a concave obstacle, as sketched in Figure 7. The inlet is at the bottom left corner, and the outlet is at the top right corner.

The simulated result for each scenario using the parameters described in the previous sections are plotted in each Sub-figure (a) of Figures 4 to 7 . In these figures, the regions filled with arrows represent the road area which is available for the vehicle, and is also the domain for flow field calculation. The cyan blocks stand for the obstacles. Inlet and outlet are marked red and yellow respectively. Green arrows are the velocity vectors of the flow field. Purple curves depict the path of the simulated vehicle, and related blue rectangles show the border of the vehicle with a certain interval. Filled contours in the fluid domain represent the divergency value.

From Sub-figure (a) for each scenario, it can be seen that the proposed method succeeds in guiding the vehicle to complete its task in a smooth path. 

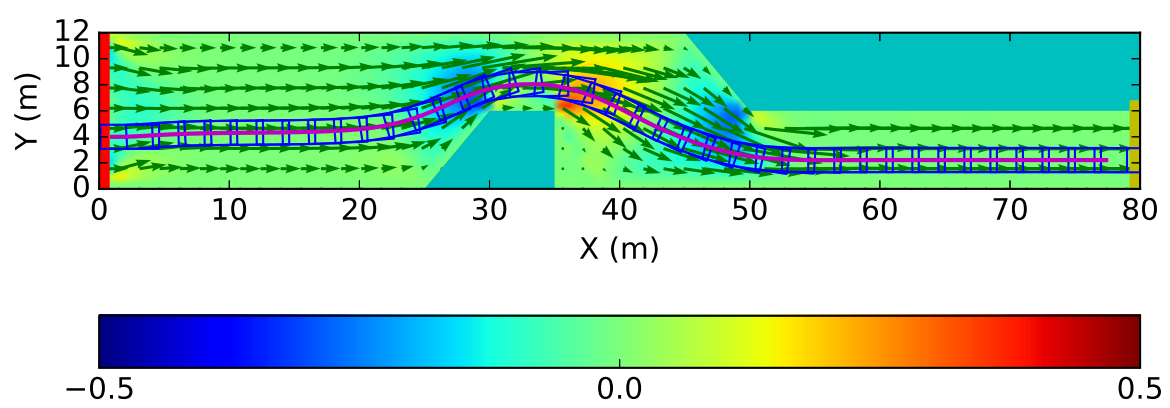

(a) $10^{-5} \mathrm{~m} / \mathrm{s}$, wall friction
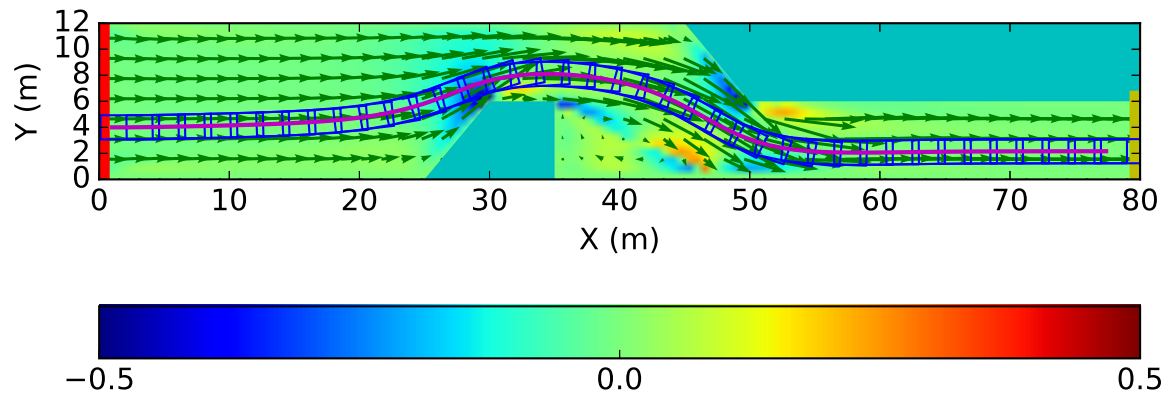

(b) $10^{-3} \mathrm{~m} / \mathrm{s}$, wall friction
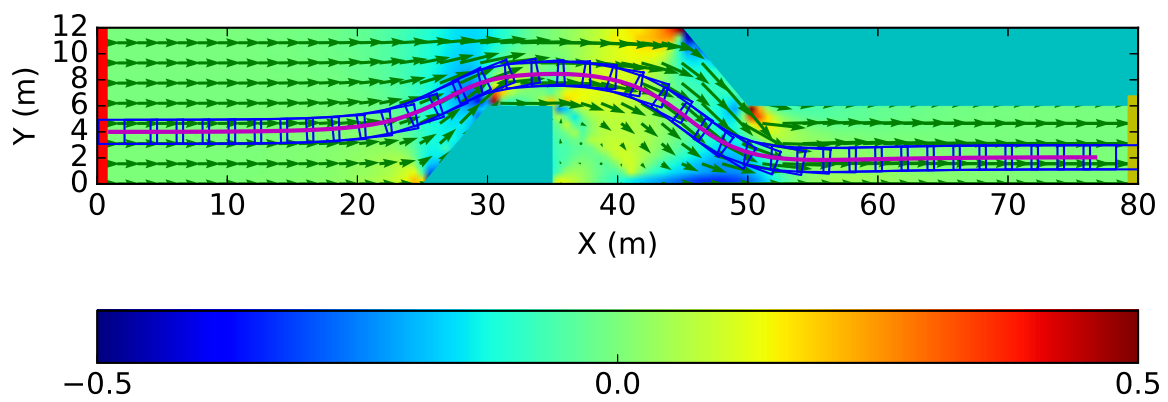

(c) $10^{-5} \mathrm{~m} / \mathrm{s}$, no wall friction

Figure 4. Velocity fields (green arrows), divergency fields (background colors) and simulated paths for different inlet speed and wall friction of Scenario 1

\subsection{Influence of CFD parameters}

In this section we try two different settings during the calculation of the flow field. The first one is to increase the inlet fluid speed from $10^{-5} \mathrm{~m} / \mathrm{s}$ to $10^{-3} \mathrm{~m} / \mathrm{s}$, whose results are plotted in each Sub-figure (b) of Figures 4 to 7 . The second one is to omit wall frictions, so that the velocity of the fluid has not to be reduced to 0 when closer to the walls. The results are plotted in each Sub-figure (c).

For Scenario 1, the emergence of a recirculation area under higher fluid speed is again seen, here in Figure $4(\mathrm{~b})$ around $(40 \mathrm{~m}, 3 \mathrm{~m})$, where some arrows point to the left. In the absence of an external disturbance, the vehicle does not enter the recirculation area. Indeed, the recirculation area has the effect of pushing the vehicle away from its location. For Figure 4 (c), omitting wall friction makes the fluid more difficult to decelerate, and more difficult to turn back after a previous turn. Due to this effect, although there is no recirculation area in the flow field, the vehicle acts similarly to the one in Figure 4 (b). 


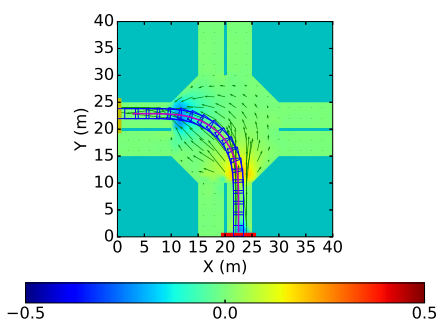

(a) $10^{-5} \mathrm{~m} / \mathrm{s}$, wall friction

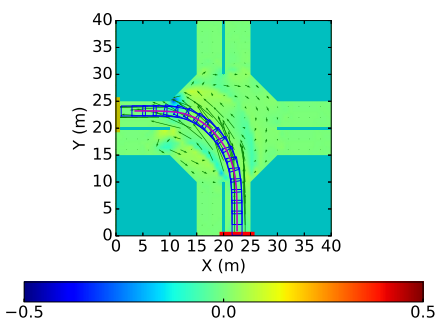

(b) $10^{-3} \mathrm{~m} / \mathrm{s}$, wall friction

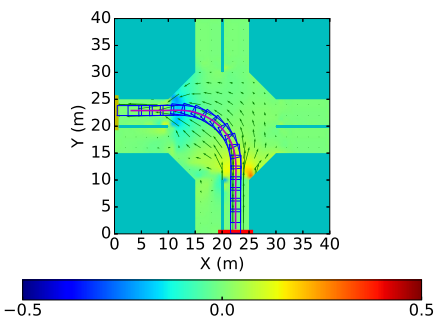

(c) $10^{-5} \mathrm{~m} / \mathrm{s}$, no wall friction

Figure 5. Velocity fields (green arrows), divergency fields (background colors) and simulated paths for different inlet speed and wall friction of Scenario 2

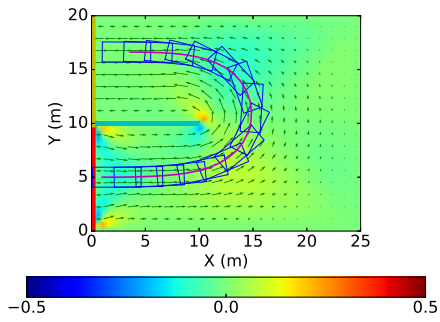

(a) $10^{-5} \mathrm{~m} / \mathrm{s}$, wall friction

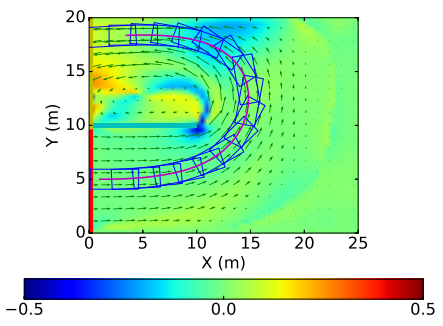

(b) $10^{-3} \mathrm{~m} / \mathrm{s}$, wall friction

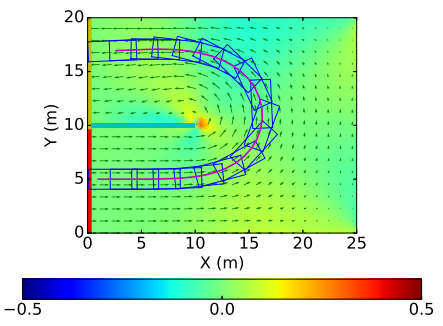

(c) $10^{-5} \mathrm{~m} / \mathrm{s}$, no wall friction

Figure 6. Velocity fields (green arrows), divergency fields (background colors) and simulated paths for different inlet speed and wall friction of Scenario 3

The filled contours represent the divergency field. In Figure 4 (a), fluid is converging before the obstacle around $(28 \mathrm{~m}, 6 \mathrm{~m})$, and diverging after it. The vehicle has gone through the region of high divergency around $(37 \mathrm{~m}, 6 \mathrm{~m})$. High divergency literally offers poor guidance, since it provides inconsistent direction. Fortunately this is not a branching situation, the vehicle will eventually turn to the correct direction just by following the result of the least squares method. When the inlet speed of the fluid is high, as shown in Figure 4 (b), the pushing of the recirculation area makes the flow outside the recirculation area more convergent, which is actually beneficial from the perspective of the divergency field. In Figure 4 (c), the absence of wall friction reduces the fluid's ability to spread the information of obstacles, making the converging and diverging region closer to the boundaries and corners. Hence the flow quality for fitting the rigid vehicle motion is somewhat improved.

For Scenario 2, the roads towards $Y+$ and $X+$ do not lead to an outlet. Therefore the fluid in these regions are almost stationary, acting like obstacles and preventing the vehicle from entering these regions. The fluid and the guided vehicle have no choice but to turn left towards the outlet. In Figure 5 (b), two recirculation areas can be found around $(15 \mathrm{~m}, 15 \mathrm{~m})$ and $(25 \mathrm{~m}, 25 \mathrm{~m})$. But it does not influence the outcome of this scenario noticeably. The removal of wall friction also has little influence in this scenario.

The divergency fields are also plotted as filled contours in Figure 5. Characteristics similar to Scenario 1 can be seen by comparing the sub-figures. However it does not lead to much difference in the simulated path.

For Scenario 3, the u-turn situation provides a large free space for the vehicle to move in, and the influences of changing the fluid parameters are easier to observe. In Figure 6 (b), a large recirculation area is located around $X$ at $0 \sim 10 \mathrm{~m}, Y$ at $10 \sim 13 \mathrm{~m}$, pushing the vehicle extremely close to the upper boundary. Actually, the CFD iteration does not converge for this settings, because the recirculation area causes reverse flow on the outlet 


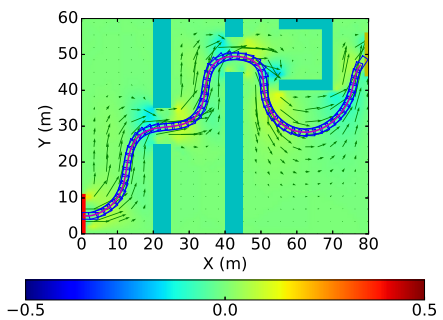

(a) $10^{-5} \mathrm{~m} / \mathrm{s}$, wall friction

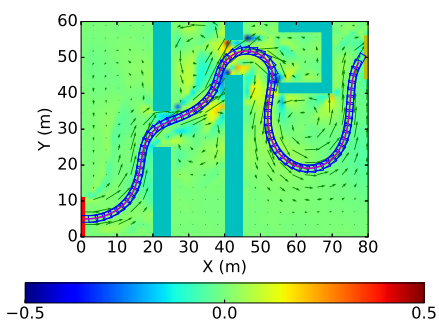

(b) $10^{-3} \mathrm{~m} / \mathrm{s}$, wall friction

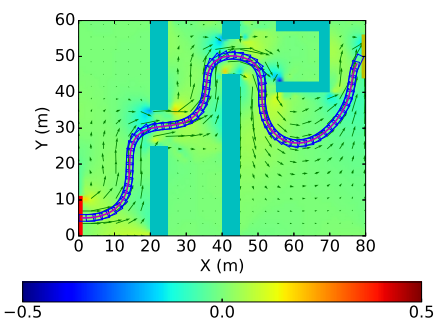

(c) $10^{-5} \mathrm{~m} / \mathrm{s}$, no wall friction

Figure 7. Velocity fields (green arrows), divergency fields (background colors) and simulated paths for different inlet speed and wall friction of Scenario 4

boundary. By comparing Figure 6 (a) and Figure 6 (c), it is noticed that omitting wall frictions barely influences the simulated path in this case.

By comparing Figure 6 (a) and (c), a noticeable converging region can be found near the inlet when wall friction exists. However the simulated path does not differ from each other remarkably, as long as the vehicle starts from the center of the converging region. For the high fluid speed setting, as shown in Figure 6 (b), multiple diverging regions can be found not only in the upper part of the domain, but also on the right side of the domain. Diverging regions would give the vehicle inconsistent guidance, therefore, in this scenario, flow field with high inlet fluid speed is less acceptable.

For Scenario 4, as shown by the three sub-figures in Figure 7, the vehicle is not trapped in the concave obstacle located around $(65 \mathrm{~m}, 50 \mathrm{~m})$, which is one of the advantages of the proposed method. Multiple recirculation areas can be seen in Figure 7 (b), some of which almost push the vehicle into collision with the obstacles. Although it reaches the outlet, the path in Sub-figure (b) is remarkably more dangerous than the one in Sub-figure (a), since the simulated path is closer to the walls. Similarly, the change of wall frictions does not influence the outcome noticeably.

Similar to Scenario 1, both the recirculation areas caused by high inlet speed and the absence of friction reduces the divergency outside the recirculation areas, and meanwhile increase it near the obstacles.

The values of average divergency of all the flow fields are listed in Table 1, along with their numbers of iterations during the CFD calculation. The advantages and disadvantages of these three settings are:

- $10^{-5} \mathrm{~m} / \mathrm{s}$, wall friction

- Advantage: has the lowest average divergency in most scenarios; cost the least computations during the CFD calculation.

- Disadvantage: has high average divergency in some scenarios; the vehicle is more likely to go through regions with high divergency.

- $10^{-3} \mathrm{~m} / \mathrm{s}$, wall friction

- Advantage: recirculation areas can push the vehicle away from obstacles; the divergency along the vehicle's path is low.

- Disadvantage: recirculation areas are potential traps for the vehicle; vehicle's path could be dangerous due to the pushing of the recirculation areas; the CFD iteration may not converge in some scenarios.

- $10^{-5} \mathrm{~m} / \mathrm{s}$, no wall friction

- Advantage: the regions far from the obstacles have low divergency.

- Disadvantage: reduces the fluid's ability of spreading the information of the obstacles to further region; cost the most computations during the CFD calculation. 
Table 1. Information about the flow fields

\begin{tabular}{cccc}
\hline Scenario & Settings & Average Divergency & Iterations \\
\hline \multirow{2}{*}{1} & $10^{-5} \mathrm{~m} / \mathrm{s}$, wall friction & 0.034 & 92 \\
& $10^{-3} \mathrm{~m} / \mathrm{s}$, wall friction & 0.030 & 180 \\
& $10^{-5} \mathrm{~m} / \mathrm{s}$, no wall friction & 0.036 & 289 \\
\hline \multirow{2}{*}{2} & $10^{-5} \mathrm{~m} / \mathrm{s}$, wall friction & 0.022 & 85 \\
& $10^{-3} \mathrm{~m} / \mathrm{s}$, wall friction & 0.014 & 339 \\
& $10^{-5} \mathrm{~m} / \mathrm{s}$, no wall friction & 0.022 & 189 \\
\hline \multirow{3}{*}{3} & $10^{-5} \mathrm{~m} / \mathrm{s}$, wall friction & 0.025 & 106 \\
& $10^{-3} \mathrm{~m} / \mathrm{s}$, wall friction & 0.034 & - \\
\hline \multirow{2}{*}{4} & $10^{-5} \mathrm{~m} / \mathrm{s}$, no wall friction & 0.028 & 107 \\
& $10^{-5} \mathrm{~m} / \mathrm{s}$, wall friction & 0.017 & 495 \\
& $10^{-3} \mathrm{~m} / \mathrm{s}$, wall friction & 0.023 & 305 \\
\hline
\end{tabular}

According to the results and discussions, it can be concluded that using high speed for inlet fluid is inappropriate. The change of wall frictions has no obvious influence on the outcome in the tested scenarios. Its advantages and disadvantages should be further studied in more realistic and complicated circumstances. In the remainder of this paper, the setting is used with inlet speed at $10^{-5} \mathrm{~m} / \mathrm{s}$ and wall friction included.

\subsection{Variations in starting position}

As mentioned in previous sections, the vectors of the flow field act as a collection of ubiquitous signposts throughout the domain. The vehicle is guided not by a fixed preplanned path, but the flow field in its vicinity. The natural concentration of fluid near the outlet is expected to guide the vehicle back to its target when it is initially deviated from the desired course.

In Scenario 1, the inlet covers the entire left boundary from $Y=0 \mathrm{~m}$ to $12 \mathrm{~m}$. The initial position of the vehicle are now deviated laterally. Simulations when the vehicle starts at $Y=4 \mathrm{~m}, 6 \mathrm{~m}$ and $8 \mathrm{~m}$ are performed respectively. The results are plotted in Figure 8. For these different starting positions, despite that the vehicle reaches different portions of the outlet region, the flow field has guided the vehicle safely and smoothly to the designated destination.

More examples are shown with Scenario 3, as depicted in Figure 9. Sub-figures (b) and (c) are the results with different lateral deviations on the starting position. Sub-figures (d) and (e) demonstrate the results with different starting orientations. A more extreme situation is shown in Sub-figure (f), where the initial state is not only far from the inlet, but also with a wrong orientation. It is seen that the proposed method succeeds in all situations.

From the results in this section, it can be concluded that the flow field needs only to respect the environment and the pre-defined entrance and exit. The CPU time costs of CFD iterations for Scenarios 1 to 3 are $5.06 \mathrm{~s}, 4.09 \mathrm{~s}$ and $8.97 \mathrm{~s}$, respectively. Although the calculation of the flow field is time consuming, it can be done in advance and stored as an enhanced map. Taking the intersection scenario as an example, as shown in Figure 10, three flow fields representing turning left, going straight and turning right are precalculated. A vehicle that needs to use this intersection will only need to pick one of the flow fields and then follow it.

From Figure 10 (b) we can observe a small drawback of the presented method. As we use the right-hand traffic rule, the usual way that a human driver will do is to go along a straight line on the right half of the intersection. However the boundary condition of 


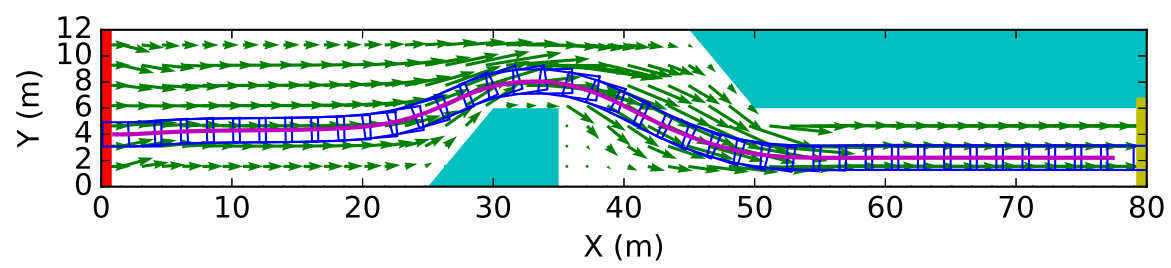

(a) $(1 \mathrm{~m}, 4 \mathrm{~m})$

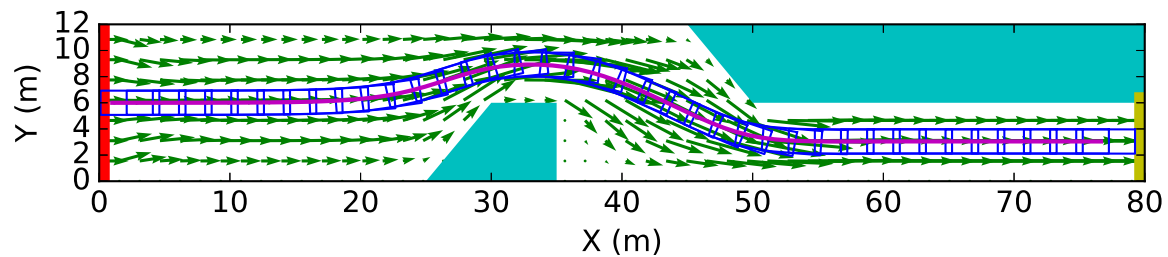

(b) $(1 \mathrm{~m}, 6 \mathrm{~m})$

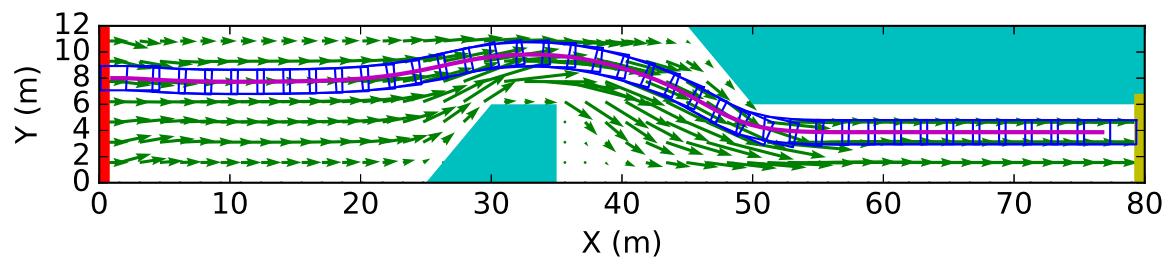

(c) $(1 \mathrm{~m}, 8 \mathrm{~m})$

Figure 8. Results for different starting positions of Scenario 1

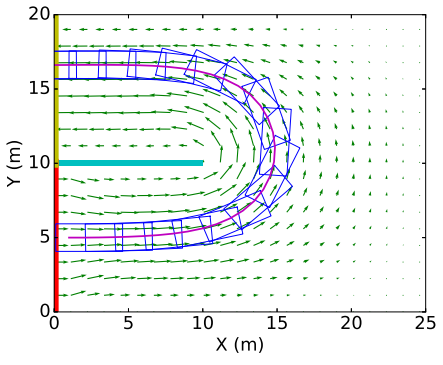

(a) $(1 \mathrm{~m}, 5 \mathrm{~m}) \quad 0^{\circ}$

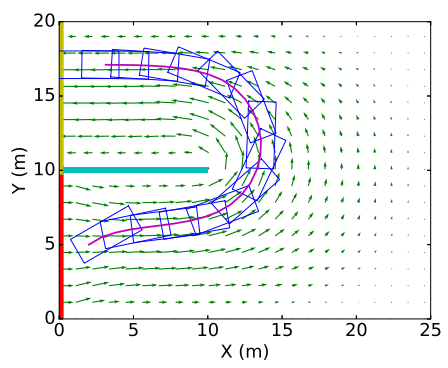

(d) $(2 \mathrm{~m}, 5 \mathrm{~m}) \quad 30^{\circ}$

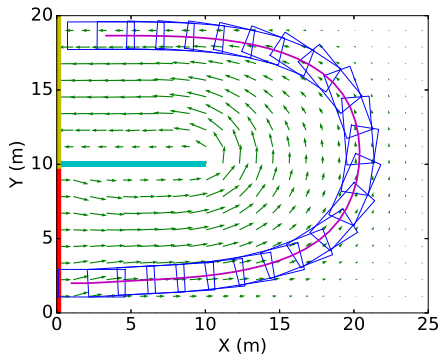

(b) $(1 \mathrm{~m}, 2 \mathrm{~m}) \quad 0^{\circ}$

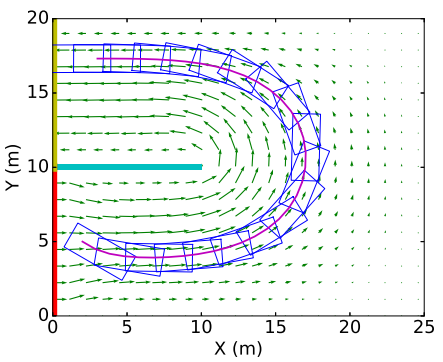

(e) $(2 \mathrm{~m}, 5 \mathrm{~m}) \quad-30^{\circ}$

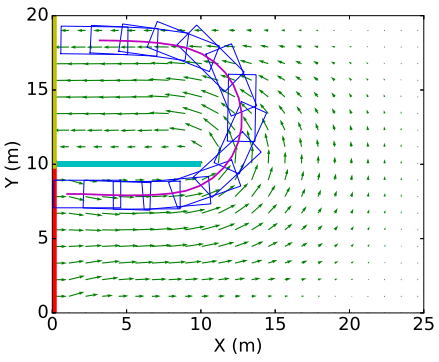

(c) $(1 \mathrm{~m}, 8 \mathrm{~m}) \quad 0^{\circ}$

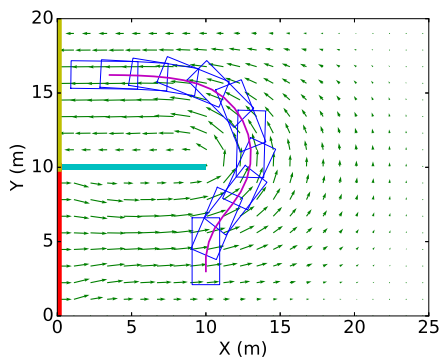

(f) $(10 \mathrm{~m}, 3 \mathrm{~m}) \quad 90^{\circ}$

Figure 9. Results for different starting positions of Scenario 3 


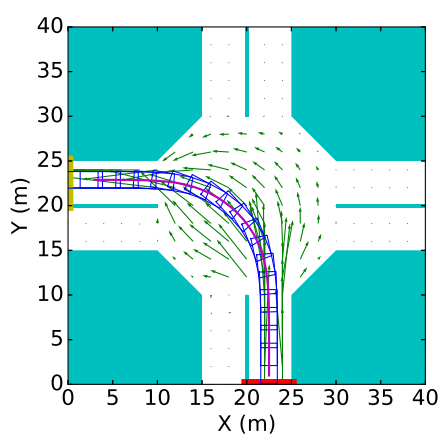

(a) Turn left

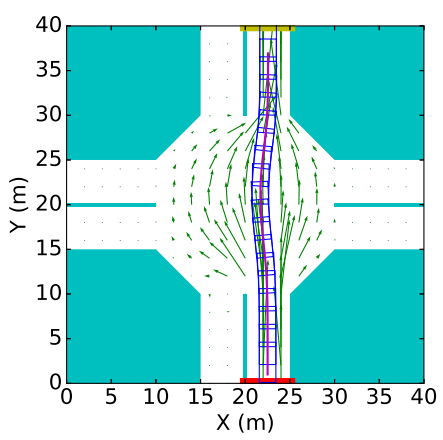

(b) Go straight

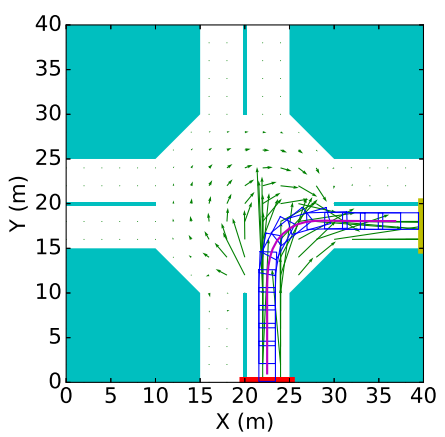

(c) Turn right

Figure 10. Results for different choices of turn of Scenario 2

the fluid is not symmetric with respect to the intersection. The fluid entering from the right-hand lane discovers larger space on its left side, causing the streamlines bend more to the left than to the right. The vehicle guided by the fluid flow then does not choose the shortest path, but also bend a little to the left.

\subsection{Branching problem}

A branching flow will typically occur when the fluid flows around both sides of a localised obstacle, and can cause critical issues for vehicle guidance. Figure 11(a) shows such a branching scenario with symmetric geometry - borders and obstacles are precisely symmetric about the $X$ axis. The calculated flow field shares this precise symmetry. If the vehicle is located on and aligned with the symmetric axis, the proposed method will not turn the vehicle and collision is inevitable. This problem can be solved either in the flow field level or in the steering control level. If the branches of the diverging flow lead to different destinations, we can simply shut down the undesired outlets when calculating the flow field, leaving only one choice for the vehicle. As we do in the intersection scenario in Figure 10, only the desired outlet is open. But if both branches are equally acceptable, a process of "choosing which branch to take" is required in the control algorithm.

The essential capabilities to deal with the branching problem in the control algorithm are: (1) knowing that the vehicle is in a diverging situation based on the flow field in the vicinity only; (2) deciding which way to go; (3) preserving that decision in the subsequent motion control. In this paper, the following extra steps are appended to the control process to achieve these capabilities.

(1) For all the nodes in the flow field that are covered by the vehicle body, calculate their average value of divergency, denoted as $\bar{\delta}$. If the average divergency is larger than a threshold (0.05 is used in this paper), the following steps are used. Otherwise, those steps will not be executed. This step implements the "knowing" capability.

(2) Define an offset to the yaw rate as $k \bar{\delta}$, where $k$ is a tunable constant coefficient, whose value is 10 in this paper.

(3) For each node in the flow field that is covered by the vehicle body, calculate the individual $\omega_{i}$ using Equation 4. These values represent the yaw rate required by each node. Count the number of positive values and negative values among all the $\omega_{i}$. If there are more positive values, add the offset to the yaw rate obtained by the least squares method. If there are more negative values, subtract the offset from the yaw rate obtained by the least squares method. If there are equal numbers of 


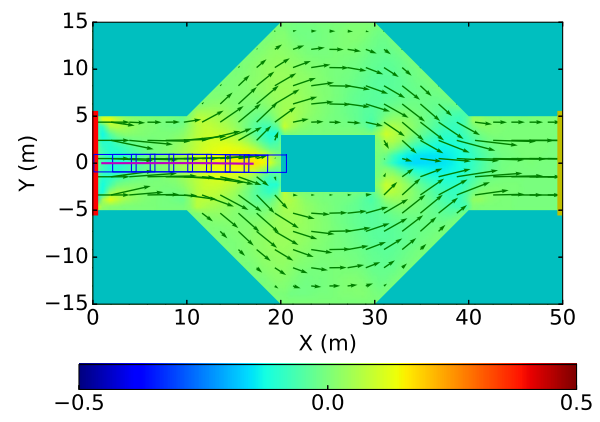

(a) Original

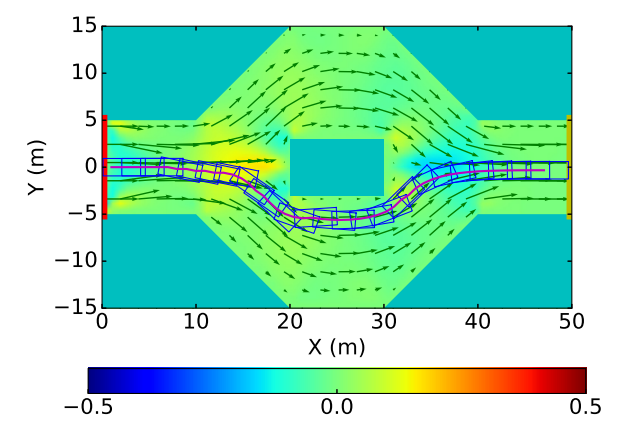

(b) With symmetry breaking

Figure 11. Results with and without the symmetry breaking procedure of Scenario 5

positive values and negative values, choose addition or subtraction randomly. This step implements the "deciding" capability.

Once the offset is applied, the vehicle will turn to the side of majority in the next control cycle. It will probably be moving towards the outside of the diverging region, and $\bar{\delta}$ will be decreased. The offset will then be decreased, and the vehicle will continue on this direction. This implements the "decision-preserving" capability.

Figure 11(b) show the results after applying these extra steps. It is seen that the vehicle not only breaks the symmetry, but also succeeds in making its way to the destination.

The above is a simple demonstration that the branching problem can be solved by involving the divergency field. The optimal values of the coefficients still need to be determined in future investigation. What the currently presented method does is driving the vehicle out of diverging flow. It has no capability of choosing the optimal branch yet. In realistic and complicated traffic circumstances, additional models and steps need to be studied before this method is ready for practical use.

\section{Conclusions}

Taking the advantage of the fluid's natural capability of path searching, this paper presents a steering control method based on guidance via fluid flow. The flow field can be pre-calculated using the CFD models, and can be made available as an enhanced digital map communicated by a "smart infrastructure". The velocity field of the fluid acts as a collection of ubiquitous signposts. The autonomous vehicle acquires the data of the fluid flow in its vicinity, and runs a steering control strategy using the simple least squares method. A divergency field is defined to evaluate the quality of the flow field. CFD calculations with different settings are tested and compared. For the branching problem, extra steps are appended to the control strategy, allowing the vehicle to choose the preferred branch, even when there is a perfectly symmetric flow. From the results and discussions, the following conclusions can be drawn.

(1) The steering control guided by a simulated flow field is feasible.

(2) Low speed laminar flow with wall friction is the preferred choice when building the guiding flow field.

(3) Although the computational time of the CFD models is not acceptable for real-time computation on board the autonomous vehicle, the flow field can be pre-calculated and stored as an enhanced digital map. The controller on the vehicle is thereby spared 
the computational cost of performing the CFD calculation. The proposed method can be practical for future automated vehicles supported by smart and connected infrastructure.

(4) The flow field can automatically pull the vehicle back on its desired course when it has been deviated. A re-planning process is not required.

(5) The divergency field is effective in evaluating the quality of the flow field in guiding the vehicle. The proposed extra steps that deal with the branching problem produce satisfactory results.

The present study focuses on low-speed scenarios. At higher speeds, the streamlines cannot be followed using the simple kinematic model presented in this paper. In that case the flow acceleration can be computed to provide a feedforward control input, while deviations in velocity can be aggregated to provide a feedback control signal. Standard techniques such as control allocation may then be applied to provide a full control structure for fluid matching at higher speeds. Overall, the methodology of using fluid flow as a guidance is still preliminary. Multiple works on this topic can be done in the future, including situations involving long vehicles, reverse driving, moving obstacles, multiple vehicles, etc.

\section{Acknowledgement}

This research is supported by the National Natural Science Foundation of China (Grant Number: 61773291).

\section{References}

[1] Dijkstra EW. A note on two problems in connection with graphs. Numerische Mathematik. 1959; (1):267-271.

[2] Stentz A. Optimal and efficient path planning for partially-known environments. In: IEEE International Conference on Robotics and Automation; 1994. p. 3310-3317.

[3] Likhachev M, Ferguson DI. Anytime dynamic A*: An anytime, replanning algorithm. In: The International Conference on Automated Planning and Scheduling (ICAPS); 2005. p. 262-271.

[4] Saffiotti A. The uses of fuzzy logic in autonomous robot navigation. Soft Computing. 1997;1(4):180 197.

[5] Sedighi KH, Ashenayi K. Autonomous local path planning for a mobile robot using a genetic algorithm. Evolutionary Computation. 2004;2:1338-1345.

[6] Phinni MJ, Sudheer A. Obstacle avoidance of a wheeled mobile robot: A genetic-neuro-fuzzy approach. In: IISc Centenary-International Conference on Advances in Mechanical Engineering; 2008.

[7] Hwang YK, Ahuja N. A potential field approach to path planning. IEEE Transactions on Robotics and Automation. 1992;8(1):23-32.

[8] LaValle SM, James J. Randomized kinodynamic planning. The International Journal of Robotics Research. 2001;20(5):378-400.

[9] Kavraki LE, Svestka P. Probabilistic roadmaps for path planning in high-dimensional configuration spaces. IEEE Transactions on Robotics and Automation. 1996;12(4):566-580.

[10] Gordon TJ, Best BC, Dixon PJ. An automated driver based on convergent vector fields. Journal of Automobile Engineering. 2002;216(4):329-347.

[11] Gordon TJ, Best MC. On the synthesis of driver control inputs for the simulation of handling manoeuvres. International Journal of Vehicle Design. 2006;40(1/2/3):52-76. 